\title{
Necrosis de un colgajo DIEP a los doce días de postoperatorio
}

\section{Twelve days postoperative necrosis of a DIEP flap}

\author{
Benito Duque, P. ${ }^{*}$, de Juan Huelves, A. ${ }^{* \star}$, Cano Rosas, M. ${ }^{*}$, Elena Sorando, E. ${ }^{* *}$
}

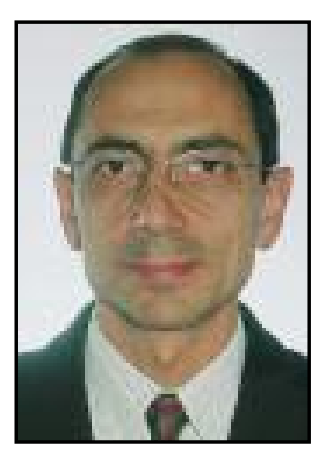

Benito Duque, $\mathrm{P}$.
Resumen

Presentamos una complicación muy poco frecuente en una paciente mastectomizada y reconstruida con un colgajo DIEP que se necrosó a los 12 días de la intervención, sin apreciarse ninguna causa precipitante. La paciente había sido sometida a radioterapia, que es una causa conocida de retraso en la neovascularización del colgajo. Consideramos que en este tipo de pacientes las medidas dirigidas a la protección del colgajo deben mantenerse durante un período de tiempo mayor que en ausencia de radioterapia.

\begin{tabular}{|l} 
Palabras clave $\quad$ Reconstrucción mamaria. Colgajo DIEP. \\
Necrosis de colgajo. \\
Código numérico 52140,158336
\end{tabular}

\begin{tabular}{|ll|}
\hline Key words & $\begin{array}{l}\text { Mammary reconstruction. DIEP-flap. } \\
\text { Flap failure. }\end{array}$ \\
Numeral Code & 52140,158336
\end{tabular}

We present an unusual complication suffered by a patient who received a DIEP flap for breast reconstruction and suffered necrosis 12 days after surgery, without existing an objective reason. Patient had been submitted to radiotherapy, that it's a known reason for delaying in neovascularización of the flap, so we consider that in this patients, measures directed to protect the flap must be extended in time, if compared with those patients without radiotherapy. 


\section{Introducción}

La reconstrucción mamaria es considerada actualmente como un paso más en el tratamiento del cáncer de mama. De entre las diversas técnicas utilizadas, los colgajos libres han ocupado un creciente interés por la calidad de sus resultados. Su supervivencia inicial depende de la integridad del pedículo, si bien la neovascularización desde los tejidos periféricos podrá mantener la viabilidad de los mismos a partir de un determinado periodo de tiempo (1).

Presentamos una paciente mastectomizada, que después de ser reconstruida con un colgajo DIEP sufrió la necrosis del mismo a los 12 días de la intervención. No pudimos objetivar una causa relacionada con dicha complicación.

\section{CASO CLÍNICO}

Se trata de una paciente de 42 años de edad, a la que se le practicó una mastectomía radical modificada por un carcinoma ductal de la mama izquierda. Como tratamiento coadyuvante recibió quimioterapia y radioterapia postoperatoriamente. Fue reconstruida un año después de la mastectomía mediante el implante de un expansor-prótesis tipo Becker, pero desarrolló progresivamente una contractura capsular grado IV de Baker a lo largo de 6 meses desde la intervención (Fig. 1).

La siguiente técnica reconstructiva consistió en la

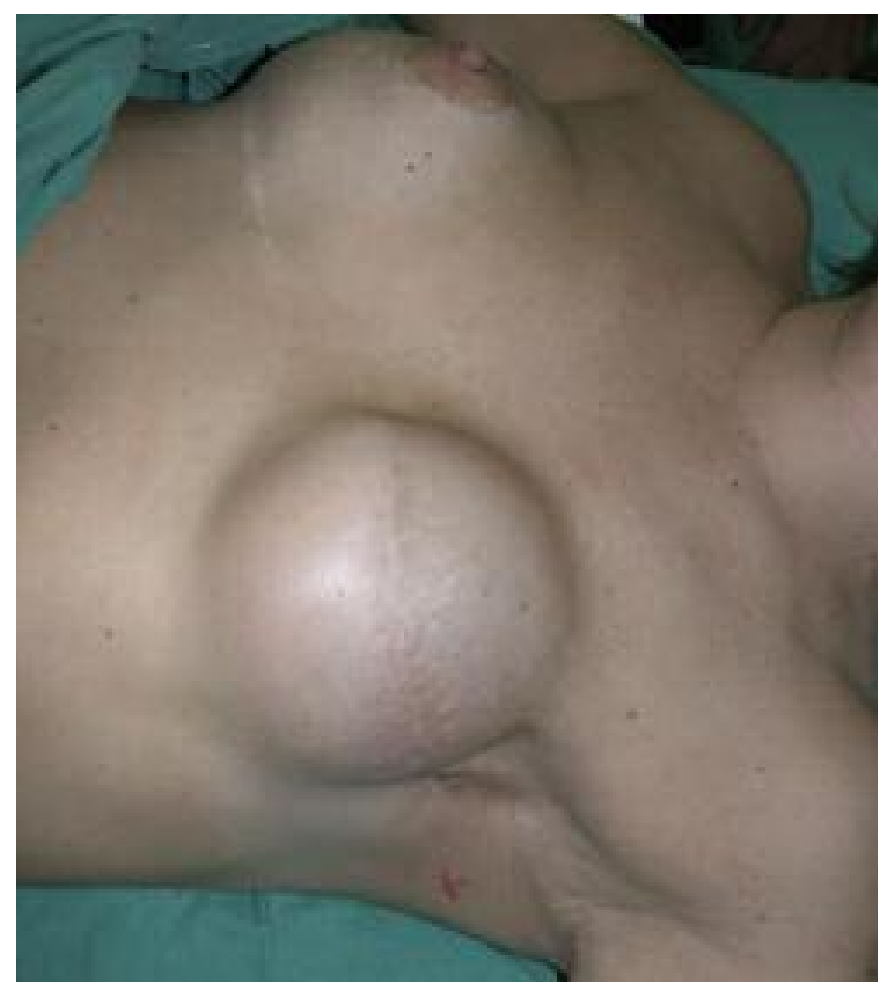

Fig. 1. Imagen preoperatoria mostrando una contractura capsular severa a los 6 meses de reconstrucción mamaria con expansor-prótesis. extracción de la prótesis de Becker y la realización de un colgajo DIEP desepitelizado anastomosado a la arteria y vena toracodorsales que se llevó a cabo a los 8 meses del implante de la prótesis de Becker. Fue monitorizado mediante observación directa a través de una pequeña ventana coincidente con el futuro emplazamiento del complejo areola-pezón (Fig. 2). La evolución postoperatoria transcurrió sin complicaciones, siendo dada el alta hospitalaria al $8^{\circ}$ día.

Durante las revisiones en consultas ambulatorias se observó a los 12 días de la intervención que el colgajo presentaba signos de isquemia a través de la ventana utilizada para su monitorización. Se procedió a la revisión quirúrgica, observando que la necrosis afectaba principalmente al área central del colgajo. La pérdida de tejido era suficientemente extensa como para obligarnos a descartar la totalidad del colgajo (Fig. 3). El colgajo DIEP fue retirado en las primeras 24 horas desde que se diagnosticó su necrosis. Establecimos una pauta de curas diarias con Sulfadiacina Argéntica y, pasada una semana se procedió a la realización de un colgajo de Latissimus Dorsi miocutaneo vascularizado por la rama del serrato, con una prótesis de gel de silicona bajo el mismo (Fig. 4, 5).

Discusión

En pacientes mastectomizadas reconstruidas con implantes, la radioterapia compromete con frecuencia

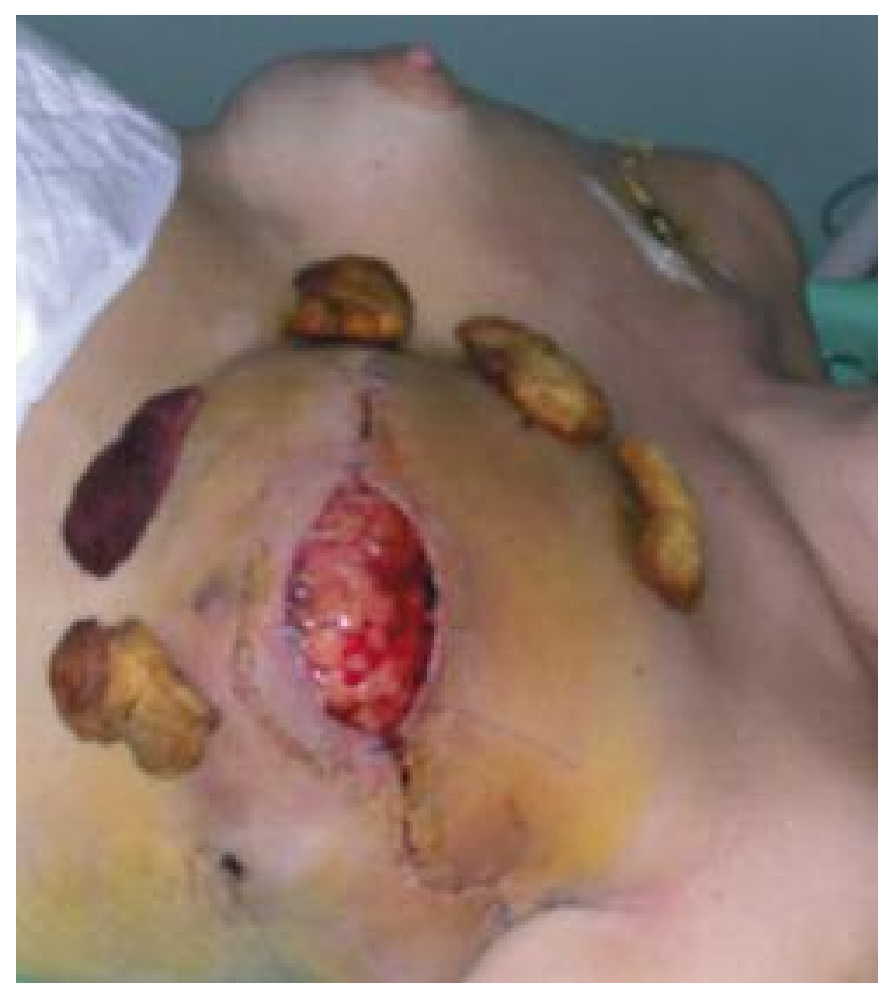

Fig. 2. Imagen del tejido de granulación visible a través de la "ventana" al $6^{\circ}$ día postoperatorio tras retirada del expansor-prótesis y reconstrucción con colgajo DIEP. 


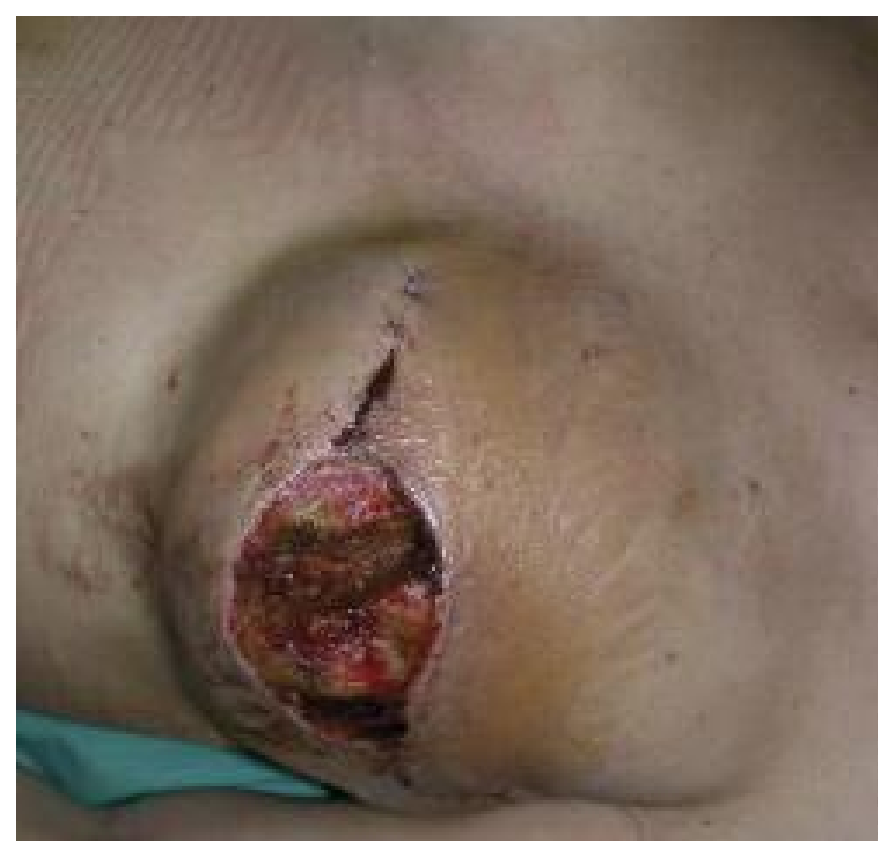

Fig. 3. Imagen a los 12 días de postoperatorio mostrando un área de necrosis central del colgajo.

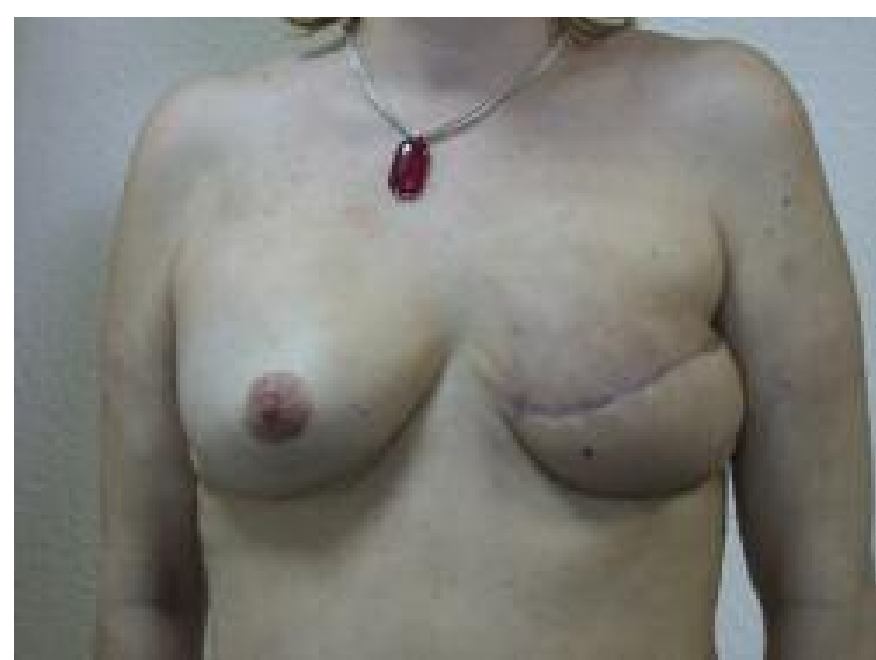

Fig. 4. Reconstrucción secundaria con colgajo de Latissimus Dorsi y prótesis redonda de gel de silicona de $200 \mathrm{cc}$. Un año de evolución.

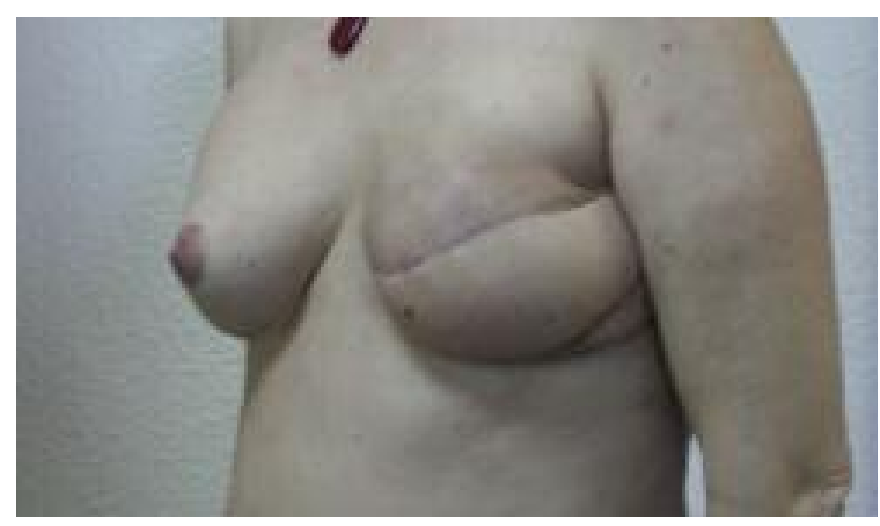

Fig. 5. Proyección oblicua.

el resultado final, por lo que los colgajos están especialmente indicados para la reconstrucción. De entre ellos, los colgajos basados en las perforantes de la arteria epigástrica profunda inferior, permiten obtener tejido adiposo-cutáneo de calidad con una mínima morbilidad (2). Los colgajos libres pueden fracasar por múltiples causas. Aunque un solo factor aislado puede ser suficiente, a menudo una serie de factores considerados menores pueden asociarse para el fallo final (1). Sin embargo, una necrosis a los 12 días es una complicación muy inusual (3-6). La paciente presentada no era fumadora ni tenía otros factores de riesgo conocidos; tampoco se observaron signos de infección. En la exploración quirúrgica durante el desbridamiento y retirada del colgajo, los restos hemáticos hallados en la zona del pedículo no ocupaban un gran espacio y aunque no sugerían corresponder a un hematoma secundario a una avulsión, tampoco se podía descartar esta posiblidad. Sí consideramos como causa más probable que una compresión, torsión o acodamiento hayan podido afectar al pedículo determinando la necrosis del colgajo $(3,4)$. Tampoco se puede descartar categóricamente la trombosis a nivel de la anastomosis, si bien, las anastomosis vasculares se supone que tienen su endotelio totalmente reestablecido hacia el cuarto día postoperatorio, por lo que es difícil explicar la obstrucción por trombosis a partir de este período.

Sabemos que el tiempo requerido para la neovascularización a partir de los tejidos circundantes se incrementa en un paciente sometido a radioterapia. Clínicamente, este periodo no se ha definido de una forma precisa. En un lecho no radiado, se ha demostrado experimentalmente que deben transcurrir al menos 8 días antes de que el pedículo vascular de un colgajo libre pueda ser dividido con seguridad (7).

Como segunda opción reconstructiva decidimos utilizar el colgajo de Latissimus Dorsi dependiente del pedículo del músculo serrato $(8,9)$. A pesar de disponer de un pedículo más corto, nos permitió elevar un colgajo miocutáneo y trasponerlo sin dificultad. Aunque se trata de una técnica descrita desde antiguo, está escasamente referida en la bibliografía. Este caso refuerza la utilidad del colgajo del latissimus Dorsi pediculado en la rama del serrato como una opción disponible en situaciones comprometidas.

\section{Conclusiones}

Es aconsejable, al realizar una reconstrucción mamaria con un colgajo libre en una paciente radiada, prevenir los movimientos y evitar la compresión externa que puedan afectar a la anastomosis, al colgajo o al pedículo durante un periodo de tiempo más prolongado.

El colgajo de Latissimus Dorsi pediculado en la rama del serrato continúa siendo una buena opción en 
cirugía reconstructiva mamaria cuando han fracasado otros métodos de primera elección.

\section{Dirección del autor}

Dr. Pablo Benito Duque

Nogales 13

Morales del Vino

49190 Zamora. España

e-mail: pablobenito@usal.es

\section{Bibliografía}

1. Black M.J.M., Chait L., O'Brien B.Mc., et al.: "How soon may the axial vessels of a surviving free flap be safely ligated?: A study in pigs". Br J Plast Surg. 1978; 31: 295.
2. Gagnon A.R., Blondeel P.N.: "Cogajos de perforantes de las arterias epigástricas inferiores profunda y superficial": . Cir. plás. iberolatinoam. 2006; 32: 243.

3. Khouri R.K.: "Avoiding free flap failure". Clin. Plast Surg. 1992;19: 773.

4. Gahankari D., Malyon A., y Weiler-Mithoff E.M.: "Avulsion of vascular anastomosis in free-flap breast reconstruction”. Br. J. Plast Surg. 2001; 54: 167.

5. Elliott L.F., Beegle P.H. y Hartrampf C.R.: "The lateral transverse thigh free flap: an alternative for autogenous-tissue breast reconstruction". Plast. Reconst. Surg. 1990; 85: 169.

6. Cavadas P.: "Late pedicle obstruction in a free Diep flap". Plast. Reconstr. Surg. 2003;112: 1199.

7. Moolenburgh S.E., van Huizum M.A. y Hofer S.O.P.: "DIEPflap failure after pedicle division three years following transfer". Br. J. Plast. Surg. 2005; 58, 1000.

8. Fisher, J., Bostwick, J., y Powell, R. W.: "Latissimus dorsi blood supply after thoracodorsal vessel division: The serratus collateral". Plast. Reconstr. Surg. 1983;72: 502.

9. Shigehara, T., Tsukagoshi, T., Satoh, K., Tatezaki, S., y Ishii, T.: "A reversed-flow latissimus dorsi musculocutaneous flap based on the serratus branch in primary shoulder reconstruction". Plast. Reconstr. Surg. 1997; 99: 566. 


\title{
Comentario al trabajo uNecrosis de un colgajo DIEP a los doce días de postoperatorio॥
}

\author{
Dr. Kevin Malouf y Dr. Phillip Blondeel \\ Departamento de Cirugía Plástica y Reconstructiva del Hospital Universitario de Gante, Bélgica.
}

El caso descrito es una presentación poco común de una necrosis a los doce días de postoperatorio. No parece haber ninguna causa y tampoco aparece ningún factor de riesgo en la paciente. El colgajo de perforante de la arteria epigástrica inferior (DIEAP) es un tejido ideal para la reconstrucción mamaria, pero al igual que cualquier otro procedimiento microquírurgico, no está exento de complicaciones. Están recogidas de un 1 a un 5\% de necrosis, parciales o totales. La forma habitual de presentación de estas necrosis, ya sean secundarias a isquemia o a congestión venosa, aparece la mayor parte de las veces en las primeras 48 horas, aunque los primeros 4 ó 5 días de postoperatorio son los más cruciales.

En el caso presentado en particular, se sustituyó un expansor tisular por un colgajo DIEP libre. En torno al expansor se desarrolla una fuerte contractura tras la radioterapia adyuvante. Debido a esta severa contractura, la cápsula tuvo que ser extirpada quirúrgicamente. A pesar de ello, a menudo notamos que la piel radiada se retrae más intensamente que el tejido sano si no están presentes otras fuentes excéntricas de intensidad suficiente. A pesar de que el bolsillo disecado sea adecuado en forma y tamaño, la capacidad retráctil de la piel puede ser infravalorada.

En otras palabras, existe una posibilidad de que el edema normal postoperatorio del colgajo, combinado con la reacción contráctil de la piel y del tejido celular subcutáneo subyacente que habitualmente aparecen tras extirpar el expansor mamario, unidos a la contractura adicional originada por la radioterapia, hayan puesto al colgajo DIEP bajo una presión constante significativa. Esta presión probablemente fue lo suficientemente fuerte para comprimir y ocluir el aporte microvascular al colgajo de una forma lenta pero progresiva. Todo ello dio lugar a una lenta muerte del colgajo, difícil de detectar por su desarrollo progresivo. Los síntomas pudieron empezar con una leve isquemia que tornaría el colgajo pálido, pero no lo suficiente como para poner sobre aviso al observador externo. Más adelante esto se traduciría en la pérdida total del tejido.

Otra causa de compresión es la presencia de bandas cicatriciales en la superficie interna de los colgajos de la mastectomía, especialmente en la parte superior y alrededor de la axila; deberían ser liberadas mediante la resección de todas las cicatrices en los bordes de los colgajos de la mastectomía y la incisión de todas las cicatrices internas, para lograr así romper la tensión contráctil en torno al colgajo. Además la parte de piel que en la mastectomía se ajusta lateralmente y queda a modo de "oreja de perro" en la línea media axilar, debe también ser reclutada para favorecer la proyección anterior de la cobertura cutánea.

En este caso, pudiera ser posible que la isquemia progresiva se desarrollara ya en el momento del alta hospitalaria o justo después y que la necrosis del colgajo no se apreciara hasta que la paciente regresó para control a los 12 días de postoperatorio.

A fin de evitar este tipo de complicaciones, es recomendable eliminar todo aquel tejido que esté dañado por la radioterapia, incluyendo la cápsula, y crear un bolsillo lo suficientemente grande para acomodar el colgajo. Se debería haber dejado una isla de piel más ancha entre el colgajo superior y el inferior de la mastectomía de manera que, en caso de aparecer un edema extenso o una excesiva contractura y/o fuerza compresiva del colgajo, hubiera suficiente piel blanda y elástica que permitiera al colgajo inflamarse y expandirse. Cualquier exceso de esa piel que pudiera provocar posteriormente ptosis mamaria, podría extirparse más adelante.

\section{Respuesta al comentario de los Drs. Malouf y Blondeel}

\section{Dr. Pablo Benito Duque}

Deseo agradecer sinceramente el interés demostrado, así como el comentario al trabajo por parte de los doctores Malouf y Blondeel, tanto por su calidad, como por los consejos prácticos que se extraen del mismo.

La pormenorizada valoración que hacen de las posibles complicaciones asociadas a la técnica utilizada, es un buen repaso de los pasos técnicos, y de las trampas a evitar en las pacientes radiadas y sometidas a reconstrucción mamaria mediante microcirugía.
Creemos que la posibilidad de un compromiso vascular del colgajo, causado por la combinación de los factores enumerados por los citados doctores, debe ser tenida en cuenta en el caso presentado. El conjunto de los mismos puede no haber sido valorado en su justa medida por nuestra parte. Una vez más vemos la importancia de ser lo más exhaustivos posible, a la hora de valorar las zonas receptoras de colgajos.

Deseo despedirme agradeciendo al comité científico la deferencia por haber tenido en consideración el trabajo presentado. 